\title{
Decoupling the momentum constraints in general relativity
}

\section{J. Tafel ${ }^{1}$}

Received: 16 January 2015 / Accepted: 22 August 2015 / Published online: 1 September 2015 (C) The Author(s) 2015. This article is published with open access at Springerlink.com

\begin{abstract}
We present a $2+1$ decomposition of the vacuum initial conditions in general relativity. For a constant mean curvature one of the momentum constraints decouples in quasi isotropic coordinates and it can be solved by quadrature. The remaining momentum constraints are written in the form of the tangential Cauchy-Riemann equation. Under additional assumptions its solutions can be written in terms of integrals of known functions. We show how to obtain initial data with a marginally outer trapped surface. A generalization of the Kerr data is presented.
\end{abstract}

Keywords Initial constraints · Conformal method $\cdot$ Marginally trapped surfaces

\section{Introduction}

Vacuum initial data in general relativity consist of a Riemannian metric $\tilde{g}=\tilde{g}_{i j} d x^{i} d x^{j}$ and a symmetric tensor $\tilde{K}=\tilde{K}_{i j} d x^{i} d x^{j}$ given on a 3 -dimensional manifold $S$. These data have to satisfy the constraint equations

$$
\begin{aligned}
\tilde{\nabla}_{j}\left(\tilde{K}^{j}{ }_{i}-\tilde{H} \delta^{j}{ }_{i}\right) & =0 \\
\tilde{R}+\tilde{H}^{2}-\tilde{K}_{i j} \tilde{K}^{i j} & =0,
\end{aligned}
$$

where $\tilde{\nabla}_{i}$ are covariant derivatives corresponding to $\tilde{g}, \tilde{R}$ is the Ricci scalar of $\tilde{g}$ and $\tilde{H}=\tilde{K}_{i}{ }_{i}$. Tensors $\tilde{g}$ and $\tilde{K}$ are interpreted, respectively, as the induced metric and

J. Tafel

tafel@fuw.edu.pl

1 Institute of Theoretical Physics, University of Warsaw, Hoża 69, 00-681 Warsaw, Poland 
the external curvature of $S$ embedded in a 4-dimensional spacetime developing from these data in accordance with the Einstein equations.

The conformal approach to the constraints of Lichnerowicz, Choquet-Bruhat and York (see [1-3] for a review) is based on the following representation of initial data

$$
\tilde{g}_{i j}=\psi^{4} g_{i j}, \quad \tilde{K}_{i j}=\psi^{-2} K_{i j}+\frac{1}{3} \tilde{H} \psi^{4} g_{i j}
$$

where $H=K^{i}{ }_{i}=0$. The momentum constraint (1) yields equations for $g_{i j}, K_{i j}, \tilde{H}$ and $\psi$ with no derivatives of $\psi$, whereas the Hamiltonian constraint (2) is equivalent to the Lichnerowicz equation

$$
\Delta \psi=\frac{1}{8} R \psi-\frac{1}{8} K_{i j} K^{i j} \psi^{-7}+\frac{1}{12} \tilde{H}^{2} \psi^{5}
$$

where $\Delta$ and $R$ are, respectively, the covariant Laplace operator and the Ricci scalar of metric $g$.

If $\tilde{H}=$ const (constant mean curvature data) the momentum constraint (1) is equivalent to

$$
\nabla_{j} K_{i}^{j}=0, \quad K_{i}^{i}=0 .
$$

In this case one can first find a solution $(g, K)$ of (5) and then consider Eq. (4) for $\psi$. Equation (5) were solved analytically or reduced to a simpler system only for conformally flat initial metrics [4-7] or symmetric data [8-12]. Exact solutions of (4) are known for the simplest class of data with $K=0$ and conformally flat $g$. In other cases, the best what one can do is to prove the existence of solutions [13-16] or to find them numerically (see $[17,18]$ for a review).

The aim of this paper is to reduce Eq. (5) to a simpler system. We show that these equations decouple in coordinates closely related to quasi isotropic coordinates of Smarr [20]. One of the equations yields a component $W$ of $K$ as an integral of free data. Then the remaining two equations can be written as a single complex equation using the Cauchy-Riemann structure related to the metric $g$. Under additional assumptions solutions of this equation can be also represented as integrals of known functions.

In the last section we consider data admitting a marginally outer trapped surface (MOTS) which can be considered as an attribute of a black hole. Known constructions of such data are based on the puncture method of Brill and Lindquist [4], the conformal-imaging method of Misner [5] or the boundary condition method proposed by Thornburg [21]. In the spirit of Misner's approach we define a class of maximal nonconformally flat data with a reflection symmetry which assures existence of MOTS. These data generalize the Kerr metric data but they don't have to be axially symmetric.

\section{The $2+1$ decomposition of initial data}

We would like to find coordinates in which the momentum constraint (5) decouples and can be partly integrated. Let us foliate the initial manifold $S$ into surfaces given 
by constant levels of a function $\varphi$. In coordinates $x^{i}=x^{a}, \varphi$, where $i=1,2,3$ and $a=1,2$, an arbitrary metric $g$ on $S$ can be written in the form

$$
g=g_{a b} d x^{a} d x^{b}+\alpha^{2}\left(d \varphi+\beta_{a} d x^{a}\right)^{2}
$$

Coordinate transformations allow to impose up to 3 conditions on components of this metric. Most of them, including conditions satisfied in the Gauss coordinates, do not simplify Eq. (5). The method of trial and error shows that probably the best coordinates in this respect are $x^{a}, \varphi$ such that the 2-dimensional metric $g_{a b}$ is conformally flat

$$
g=\rho^{2} \delta_{a b} d x^{a} d x^{b}+\alpha^{2}\left(d \varphi+\beta_{a} d x^{a}\right)^{2} .
$$

In order to find these coordinates one has to solve equation

$$
\xi_{, i} \xi^{, i}=0
$$

for a complex function $\xi$ such that $d \xi \wedge d \bar{\xi} \wedge d \varphi \neq 0$. Then $\operatorname{Re} \xi, \operatorname{Im} \xi$ and $\varphi$ are new coordinates in which metric takes the form (7). Still one can change coordinate $\varphi$. This freedom allows to reduce a number of functions in $g$ to three. For instance, in this way one can obtain conditions satisfied by the quasi isotropic coordinates [20].

We are not able to describe all initial metrics which admit solutions of (8). Throughout the paper we will assume existence of coordinates $x^{a}, \varphi$ such that $g$ is given by (7). It is not very strong restriction compared to the assumption $\tilde{H}=$ const. We will work in a single coordinate system. Given a solution of (5) one can try to extend it to an acceptable initial manifold. Coordinates $x^{i}$ may be analogs of the Cartesian coordinates in flat space but they may be also related to spherical-like coordinates with a radial distance defined by $x^{1}$ and angles $x^{2}=\theta$ and $\varphi$. In the latter case initial data should be periodic in $\varphi$ and satisfy appropriate conditions at $\theta=0, \pi$ (see Sect. 3).

Let us choose the following basis of 1 -forms and the dual vector basis

$$
\begin{aligned}
& \theta^{a}=d x^{a}, \quad \theta^{3}=d \varphi+\beta_{a} d x^{a} \\
& e_{a}=\partial_{a}-\beta_{a} \partial_{\varphi}, \quad e_{3}=\partial_{\varphi}
\end{aligned}
$$

adapted to metric (7). It is convenient to define a complex coordinate $\xi$, a complex operator $\partial$ and functions $\beta, U, V, W$ as follows

$$
\begin{aligned}
\xi & =x^{1}+i x^{2}, \quad \beta=\frac{1}{2}\left(\beta_{1}-i \beta_{2}\right), \quad \partial=\partial_{\xi}-\beta \partial_{\varphi} \\
U & =\frac{1}{2} \alpha\left(K_{11}-K_{22}\right)-i \alpha K_{12}, \quad V=\alpha\left(K_{13}-i K_{23}\right), \quad W=K_{a}^{a} .
\end{aligned}
$$

In coordinates $\xi, \bar{\xi}, \varphi$ metric $g$ reads

$$
g=\rho^{2} d \xi d \bar{\xi}+\alpha^{2}(d \varphi+\beta d \xi+\bar{\beta} d \bar{\xi})^{2}
$$


Proposition 2.1 The momentum constraint (5) with metric (13) decouples into the following system of equations for a real function $W$ and a complex function $U$

$$
\begin{aligned}
& \left(\rho^{3} W\right)_{, \varphi}=E \\
& U_{, \bar{\xi}}-(\bar{\beta} U)_{, \varphi}=F,
\end{aligned}
$$

where $E$ is given by (21) and $F$ is given up to $W$ by (22). Free data consist of metric $g$, complex function $V$, a real $\varphi$-independent function $W_{0}$ and a complex function $U_{0}$ satisfying

$$
U_{0, \bar{\xi}}-\left(\bar{\beta} U_{0}\right)_{, \varphi}=0
$$

Proof Let $\hat{K}_{a}{ }^{b}$ be the traceless part of $K_{a}{ }^{b}$

$$
K_{a}^{b}=\hat{K}_{a}^{b}+\frac{1}{2} W \delta_{a}^{b}
$$

In basis (10) the momentum constraint (5) with $i=3$ yields (14) with

$$
E=\rho \alpha^{-1} e_{a}\left(\alpha \rho^{2} K_{3}^{a}\right)-2 \rho^{3} \beta_{a, \varphi} K_{3}^{a} .
$$

For $i=a$ one obtains

$$
\rho^{2}\left(\alpha \hat{K}_{a}^{b}\right)_{\mid b}-\left(\alpha \rho^{2} \hat{K}_{a}^{b} \beta_{b}\right)_{, \varphi}=F_{a},
$$

where ${ }_{\mid b}$ denotes the covariant derivative with respect to metric $g_{a b}=\rho^{2} \delta_{a b}$ and

$$
F_{a}=-\frac{1}{2} \rho^{2} \alpha^{-2} e_{a}\left(\alpha^{3} W\right)+\frac{3}{2} \rho^{2} \alpha W \beta_{a, \varphi}+\rho^{2} \alpha\left(\eta^{c d} e_{c} \beta_{d}\right) \eta_{a b} K_{3}^{b}-\left(\alpha^{-1} \rho^{2} K_{3 a}\right)_{, \varphi} .
$$

By means of (11) and (12) formula (18) can be written as

$$
E=2 \rho \alpha^{-1} \operatorname{Re}\left(\bar{\partial} V-2 \bar{\beta}_{, \varphi} V\right) .
$$

A complex combination of Eq. (19) with $a=1$ and $a=2$ leads to (15) with $F=$ $\frac{1}{2}\left(F_{1}-i F_{2}\right)$ of the form

$$
F=-\frac{1}{2} \rho^{2} \alpha^{-2} \partial\left(\alpha^{3} W\right)+\frac{3}{2} \rho^{2} \alpha W \beta_{, \varphi}-2 i \operatorname{Im}(\bar{\partial} \beta) V-\frac{1}{2}\left(\alpha^{-2} \rho^{2} V\right)_{, \varphi} .
$$

Equation (14) determines $W$ up to a real function $f(\xi, \bar{\xi})$

$$
W=\rho^{-3}\left(\int_{\varphi_{0}}^{\varphi} E d \varphi^{\prime}+f(\xi, \bar{\xi})\right) .
$$


Function $f$ is in one to one correspondence to function $W_{0}=\left.W\right|_{\varphi=\varphi_{0}}$. Substituting (23) into (22) yields $F$ up to $f$. Then (15) becomes an equation for $U$. Its solution, if it exists, is defined up to a solution $U_{0}$ of the homogeneous part of (15).

Note that Eqs. (14)-(15) are invariant under transformation (3) with $\tilde{H}=0$ since (5) is invariant. Using this freedom one can fix one of components of $g$. Another component can be fixed by transformation of coordinate $\varphi$.

Substituting $F=\hat{F}_{, \varphi}$ into (15) leads to the equation

$$
\bar{\partial} \hat{U}=\hat{F}
$$

for a function $\hat{U}$ such that $U=\hat{U}_{, \varphi}$. Operator $\partial$ defines the Cauchy-Riemann (CR) structure (see [22] and references therein) on the initial manifold, not unique since locally there are many systems of coordinates in which metric takes the form (7). The example of Lewy [23] shows that Eq. (24) can be unsolvable for some functions $\beta$ and $\hat{F}$. CR structures are known to appear in general relativity, especially in the context of algebraically special solutions of the Einstein equations (see e.g. [24]).

There are several cases in which solutions of (24) can be represented in an integral form. In all of them the CR structure is realizable. This means that, in addition to $\xi$, there exists a solution $\chi$ of equation $\bar{\partial} \chi=0$ such that $\chi, \varphi \neq 0$. Equivalently, $\beta$ can be written in the form

$$
\beta=\frac{\bar{\chi}, \xi}{\bar{\chi}_{, \varphi}} .
$$

Given $\chi$ the initial manifold can be considered as a 3-dimensional real surface in space $C^{2}$ of pairs $(\xi, \chi)$.

Proposition 2.2 If $\beta=0$ then $U$ is given by

$$
U=\frac{1}{2 \pi i} \int_{\Omega} \frac{F\left(\xi^{\prime}, \bar{\xi}^{\prime}, \varphi\right)}{\xi^{\prime}-\xi} d \xi^{\prime} \wedge d \bar{\xi}^{\prime}+h(\xi, \varphi),
$$

where the integral is taken over a bounded open neighborhood $\Omega$ of $\xi$ in $C$ and $h$ is an arbitrary function holomorphic in $\xi$.

Proof For $\beta=0$ Eq. (15) reads

$$
U_{, \bar{\xi}}=F
$$

Integrating (27) with the fundamental solution $(\pi \xi)^{-1}$ for the Cauchy-Riemann operator $\partial_{\bar{\xi}}$ leads to a version of Cauchy's integral formula (Theorem 1.2.1 in [25]) which can be converted into (26). The domain of integration can be extended to a whole support of $F$ provided that the r. h. s. of (26) still makes sense.

Proposition 2.3 Let functions $\rho, \alpha, \chi$ and $V$ be analytic with respect to coordinate $\varphi$. Then 


$$
U=\chi, \varphi\left(\frac{1}{2 \pi i} \int_{\Omega} \frac{F\left(\xi^{\prime}, \bar{\xi}^{\prime}, \varphi\left(\xi^{\prime}, \bar{\xi}^{\prime}, \chi\right)\right)}{\xi^{\prime}-\xi} \varphi_{, \chi}\left(\xi^{\prime}, \bar{\xi}^{\prime}, \chi\right) d \xi^{\prime} \wedge d \bar{\xi}^{\prime}+h(\xi, \chi)\right),
$$

where $\chi$ depends on unprimed coordinates and $\varphi\left(\xi^{\prime}, \bar{\xi}^{\prime}, \cdot\right)$ is the inverse function to $\chi\left(\xi^{\prime}, \bar{\xi}^{\prime}, \cdot\right)$.

Proof Under assumptions of this proposition functions $W, F$ and $\hat{F}$ are also analytic in $\varphi$. Thus, we can complexify $\varphi$ and pass to coordinates $\xi$, $\bar{\xi}$ and $\chi$. In these coordinates Eq. (24) reads

$$
\hat{U}_{\overline{,} \bar{\xi}}=\hat{F}(\xi, \bar{\xi}, \varphi(\xi, \bar{\xi}, \chi))
$$

Now, we can follow (26) in order to represent $\hat{U}$ as

$$
\hat{U}=\frac{1}{2 \pi i} \int_{\Omega} \frac{\hat{F}\left(\xi^{\prime}, \bar{\xi}^{\prime}, \varphi\left(\xi^{\prime}, \bar{\xi}^{\prime}, \chi\right)\right)}{\xi^{\prime}-\xi} d \xi^{\prime} \wedge d \bar{\xi}^{\prime}+\hat{h}(\xi, \chi) .
$$

If we come back to coordinates $\xi, \bar{\xi}, \varphi$ and differentiate (30) over $\varphi$ we obtain (28) with $h=\hat{h}_{, \chi}$.

Formula (28) becomes much simpler if $\beta_{, \varphi}=0$. Then we can choose

$$
\chi=\varphi+\chi^{0}(\xi, \bar{\xi}),
$$

where $\chi_{, \bar{\xi}}^{0}=\bar{\beta}$ (this condition can be always locally solved with respect to $\chi_{0}$ ). Expression (28) takes the form

$$
U=\frac{1}{2 \pi i} \int_{\Omega} \frac{F\left(\xi^{\prime}, \bar{\xi}^{\prime}, \varphi+\chi^{0}(\xi, \bar{\xi})-\chi^{0}\left(\xi^{\prime}, \bar{\xi}^{\prime}\right)\right)}{\xi^{\prime}-\xi} d \xi^{\prime} \wedge d \bar{\xi}^{\prime}+h\left(\xi, \varphi+\chi^{0}(\xi, \bar{\xi})\right) .
$$

If all initial data are independent of $\varphi$ formula (32) reduces to

$$
U=\frac{1}{2 \pi i} \int_{\Omega} \frac{F\left(\xi^{\prime}, \bar{\xi}^{\prime}\right)}{\xi^{\prime}-\xi} d \xi^{\prime} \wedge d \bar{\xi}^{\prime}+h(\xi) .
$$

In this case Eq. (14) is replaced by condition $E=0$. It implies

$$
K_{3}{ }^{a}=\alpha^{-1} \eta^{a b} \omega_{, b},
$$

where $\omega$ is a function of coordinates $x^{a}$ [12] (a potential equivalent to $\omega$ was also introduced in [9-11]). Now $F$ is determined by metric $g$ and functions $W$ and $\omega$. 
If $\chi$ and $\hat{F}$ are analytic in all coordinates then one can solve (24) by integrating (29) over $\bar{\xi}$. Then, instead of (28) one obtains

$$
U=\chi, \varphi\left(\int_{\bar{\xi}_{0}}^{\bar{\xi}} F\left(\xi, \bar{\xi}^{\prime}, \varphi\left(\xi, \bar{\xi}^{\prime}, \chi\right) \varphi, \chi\left(\xi, \bar{\xi}^{\prime}, \chi\right) d \bar{\xi}^{\prime}+h(\xi, \chi)\right)\right.
$$

This formula becomes particularly simple if data is independent of $\varphi$

$$
U=\int_{\bar{\xi}_{0}}^{\bar{\xi}} F\left(\xi, \bar{\xi}^{\prime}\right) d \bar{\xi}^{\prime}+h(\xi)
$$

All considerations in this section are purely local. Assume that we can extend local solutions of the momentum constraint (5) to a whole initial manifold, e. g. to an asymptotically Euclidean manifold. In this case the mean curvature $\tilde{H}$ must vanish. In order to complete the construction of initial data one should solve the Lichnerowicz equation (4) for the conformal factor $\psi$. Existence of a solution is equivalent to positivity of the Yamabe type invariant $[15,16]$, but there is no practical device how to satisfy the latter condition. For some classes of data one can get it from the Sobolev inequalities admitted by the initial manifold [12]. The simplest way to assure existence and uniqueness of $\psi$ is to assume that the initial manifold is complete and $R \geq 0$. For instance, one can take the same initial manifold and metric $g$ as in a known maximal $(\tilde{H}=0)$ asymptotically flat solution of the full set of initial conditions. Then $R \geq 0$ since the Hamiltonian constraint is satisfied by this solution. If we take as $K$ another solution of (5) with the same $g$ we can be sure that the Lichnerowicz equation can be solved but there is still problem to find new $\psi$ numerically.

We are not able to extend results of this section to data (3) with $\tilde{H} \neq$ const. Then Eq. (5) is no longer equivalent to (1). Equations (14) and (15) are replaced by

$$
\begin{aligned}
\left(\rho^{3} W\right)_{, \varphi} & =E-\frac{2}{3} \psi^{6} \rho^{3} \tilde{H}_{, \varphi} \\
U_{, \xi}-(\beta U)_{, \varphi} & =F+\frac{2}{3} \psi^{6} \rho^{2} \alpha \bar{\partial} \tilde{H}
\end{aligned}
$$

(note that $\beta, U, V$ and $W$ are preserved by transformation (3) and $\rho \rightarrow \psi^{2} \rho, \alpha \rightarrow$ $\psi^{2} \alpha$ ). Equations (37) and (38) have to be considered simultaneously with (4) except the case $\tilde{H}_{, \varphi}=0$ for which $W$ is given by (23). Existence of solutions of this system is much more difficult to prove $[3,19]$.

\section{Horizons}

In the theory of black holes it is important to construct initial data with one or more 2-dimensional surfaces which can represent horizons of black holes. This can be done within the conformal method by imposing an appropriate condition on the conformal factor $\psi$ on an internal boundary of the initial manifold [15]. This boundary becomes MOTS for initial data $(\tilde{g}, \tilde{K})$ given by (3). Existence of $\psi$ for asymptotically flat data 
depends on the positivity of the Yamabe type invariant what is even more difficult to prove than in the case of an unbounded initial manifold (see [12] for partial results). Another problem is that a continuation of initial data through this internal boundary is not assured. This problem does not appear in the inversion symmetry approach of Misner [5] which is applicable to a restricted class of data. In this section we propose a construction of data which follows the latter method.

We would like to generalize the Kerr metric data at $t=$ const, where $t$ is the BoyerLindquist time coordinate. The initial metric induced by the Kerr solution reads

$$
g=\rho^{2} \Delta^{-1} d r^{2}+\rho^{2} d \theta^{2}+\rho^{-2} \Sigma^{2} \sin ^{2} \theta d \varphi^{2},
$$

where

$$
\rho^{2}=r^{2}+a^{2} \cos ^{2} \theta, \quad \Delta=r^{2}-2 M r+a^{2}, \Sigma^{2}=\left(r^{2}+a^{2}\right)^{2}-a^{2} \Delta \sin ^{2} \theta .
$$

Metric (39) takes the form (7) in coordinates $x^{i}=\tilde{r}, \theta, \varphi$, where $\tilde{r}$ is related to $r$ by

$$
r=M+\sqrt{M^{2}-a^{2}} \cosh \tilde{r}, \quad \tilde{r} \in[-\infty, \infty] .
$$

Domains where $\tilde{r}>0$ or $\tilde{r}<0$ are asymptotically flat. They are connected by the external Kerr horizon (the Einstein-Rosen bridge) located at $\tilde{r}=0$. Only nonvanishing components of $K$ are given by (34) with [12]

$$
\omega=4 a M \rho^{-2}\left[2\left(r^{2}+a^{2}\right)+\left(r^{2}-a^{2}\right) \sin ^{2} \theta\right] \cos \theta .
$$

The Kerr initial data are analytic in coordinates $x^{i}$. Tensors $g$ and $K$ are invariant under the reflection

$$
x^{1} \rightarrow-x^{1}
$$

which corresponds to the inversion invariance of Misner. This and vanishing of $K^{11}$ at $x^{1}=0$ imply that surface $x^{1}=0$ is MOTS.

In order to generalize the Kerr data we assume that initial data are given by (3), metric $g$ has the form (13) and

$$
\begin{aligned}
& g \rightarrow g \\
& K \rightarrow \epsilon K, \quad \epsilon= \pm 1
\end{aligned}
$$

under reflection (43). Transformations (44)-(45) are equivalent to the following transformations of components of $g$ and $K$

$$
\begin{aligned}
& \alpha \rightarrow \alpha, \quad \rho \rightarrow \rho, \quad \beta \rightarrow-\bar{\beta} \\
& V \rightarrow-\epsilon \bar{V}, \quad W \rightarrow \epsilon W, \quad U \rightarrow \epsilon \bar{U} .
\end{aligned}
$$


Proposition 3.1 (i) Equations (14)-(15) are compatible with transformations (43) and (46)-(47). The same is true for formulas (23), (26), (28), (32)-(36) provided that

$$
\chi \rightarrow \bar{\chi}, \quad f \rightarrow \epsilon f, \quad h \rightarrow \epsilon \bar{h}, \quad \omega \rightarrow-\epsilon \omega .
$$

(ii) Let $g$ and $K$ satisfy (14)-(15), (46)-(47) and condition (trivial for $\epsilon=-1$ )

$$
\operatorname{Re} U=-\frac{1}{2} \alpha \rho^{2} W \text { at } x^{1}=0 .
$$

If the Lichnerowicz equation (4) admits a unique solution $\psi$ then surface $x^{1}=0$ is MOTS with respect to initial data $(\tilde{g}, \tilde{K})$ given by (3) with $\tilde{H}=0$.

Proof The part (i) of the proposition can be easily proved by considering induced transformations of functions $E$ and $F$ given by (21) and (22). The same refers to (34) and integral formulas for $W$ and $U$ in Sect. 2 if transformations (48) are taken into account.

The reflection invariance of $g$ implies that the exterior curvature of surface $x^{1}=0$ embedded in the initial manifold vanishes. For $\epsilon=-1$ function $K^{11}$ is antisymmetric with respect to $x^{1}$, hence automatically

$$
K^{i j} n_{i} n_{j}=0 \text { at } x^{1}=0
$$

where $n_{i}$ is the normal vector of the surface. For $\epsilon=1$ equality (50) is assured by (49). The Lichnerowicz equation is invariant under the transformation given by (43)-(45). If its solution $\psi$ is unique it must be also invariant. Then properties (45) and (50) are preserved by the conformal transformation (3). Hence, the exterior curvature of surface $x^{1}=0$ with respect to metric $\tilde{g}$ also vanishes. This together with equation (50) and $\tilde{H}=0$ imply zeroing of expansions of null rays emitted outward or inward from $x^{1}=0$. Hence, this surface is MOTS with respect to data $(g, K)$ and also to data $(\tilde{g}, \tilde{K})$.

We would like a MOTS to be diffeomorphic to the sphere $S_{2}$. Following the Kerr data let us assume that $x^{1}$ plays a role of a radial coordinate and surfaces $x^{1}=$ const are spheres with the angular coordinates $x^{2}=\theta$ and $\varphi$. Initial data should be periodic in $\varphi$ and regular at $\theta=0, \pi$. Let $\rho, \alpha, \beta_{a}$ and $K_{3 a}$ be invariant under the translation $\varphi \rightarrow \varphi+2 \pi$. Then functions $E$ and $F$ in Eqs. (14) and (15) are also invariant. Formula (23) defines periodic $W$ provided that

$$
\int_{0}^{2 \pi} E d \varphi=0
$$

We can achieve (51) in different ways e. g. by shifting one of the variables $\beta_{a}, K_{3 a}$ by a $\varphi$-independent function. Alternatively, one can use Eq. (14) in order to define $\rho$ (or $\alpha$ ) in terms of periodic functions $\alpha$ (or $\rho$ ), $\beta_{a}, \alpha K_{3 a}$ and $\rho^{3} W$. In this case we have 
to care about positivity of the resulting expression. In order to assure periodicity of $U$ Eq. (15) should be completed by the condition

$$
U(\varphi+2 \pi)-U(\varphi)=0
$$

which is compatible with (15). Note that all formulas (26), (28), (32), (35) define periodic $U$ under suitable assumptions on functions $\chi$ and $h$.

In order to avoid sigularities of metric (7) at $\theta=0, \pi$ we assume that $\rho \neq 0$ everywhere, $\alpha \neq 0$ if $\theta \neq 0, \pi$ and

$$
\alpha \approx \rho \sin \theta, \quad \beta_{2} \approx 0
$$

near $\theta=0, \pi$. Forms $\sin \theta d \theta, \sin ^{2} \theta d \varphi$ and tensor $d \theta^{2}+\sin ^{2} \theta d \varphi^{2}$ are nonsingular on $S_{2}$. In order to assure regularity of $K$ we assume that near $\theta=0, \pi$ components $K_{i j}$ of $K$ are proportional to the following powers of $\sin \theta$

$$
K_{i j} \sim(\sin \theta)^{i+j-2}
$$

or

$$
K_{33} \approx K_{22} \sin ^{2} \theta
$$

and remaining functions $K_{i j}$ satisfy (54). It follows from these conditions that

$$
\operatorname{Re} V \sim \sin ^{3} \theta, \quad \operatorname{Im} V \sim \sin ^{4} \theta, \quad E \sim \sin ^{2} \theta, \quad \operatorname{Re} F \sim \sin \theta
$$

near $\theta=0, \pi$ and $U$ should behave as

$$
\operatorname{Re} U \sim \sin \theta, \quad \operatorname{Im} U \sim \sin ^{2} \theta
$$

Above conditions are compatible with Eqs. (14)-(15).

MOTS has a chance to develop into the event horizon if initial data are asymptotically Euclidean. Let $r=c e^{x^{1}}$, where $c=$ const, approximates the radial distance in a flat space if $x^{1} \rightarrow \infty$. Standard asymptotical conditions (see e. g. [21]) are equivalent to

$$
\rho=r+0_{2}\left(r^{1-\epsilon}\right), \quad \alpha=r \sin \theta+0_{2}\left(r^{1-\epsilon}\right), \quad \beta_{a}=0_{2}\left(r^{-\epsilon}\right), \quad K_{i j}=0_{1}\left(r^{1-\epsilon}\right) .
$$

Here $\epsilon$ is a positive constant and we write $f=0_{k}\left(r^{l}\right)$ if derivatives of $f$ of order $n \leq k$ fall off as $r^{l-n}$ when $r \rightarrow \infty$.

According to Proposition 3.1 one can relatively easily construct initial data with MOTS assuming symmetry (45) with $\epsilon=-1$. For instance, let $g$ be the Kerr initial metric (39) in coordinates $\tilde{r}, \theta, \varphi$ and only non-vanishing components of $K$ are given by (34) with $\omega$ which is an even function of $x^{1}$. Since $R \geq 0$ the Lichnerowicz equation 
with $\tilde{H}=0$ admits a unique solution $\psi$ which is reflection symmetric. Given $\psi$ transformation (3) yields ultimate data which satisfies all initial constraints. Note that we can choose $\omega$ which tends to (42) if $x^{1}=\tilde{r} \rightarrow \infty$. Then the data approaches the Kerr data with the angular momentum $a$ if $x^{1} \rightarrow \infty$ and with the angular momentum $-a$ if $x^{1} \rightarrow-\infty$.

For $\epsilon=1$ condition (49) imposes an inconvenient constraint on integral representations of $W$ and $U$ from Sect. 2. Function $f$ in (23) cannot solve this constraint because $f$ is independent of $\varphi$. Function $h$ in integral formulas for $U$ can be used only for analytic fields. A radical way to avoid problem with (49) is to assume $W=\operatorname{Re} U=\beta=0$. Then Eq. (14) yields $V=2 i \omega_{, \xi}$ and (15) is equivalent to the following conjugate equations for $\operatorname{ImU}$

$$
(\operatorname{Im} U)_{, \bar{\xi}}=-\left(\rho^{2} \alpha^{-2} \omega_{, \xi}\right)_{, \varphi}, \quad(\operatorname{Im} U)_{, \xi}=-\left(\rho^{2} \alpha^{-2} \omega_{, \bar{\xi}}\right)_{, \varphi}
$$

The integrability condition for this system reads

$$
\left(\rho^{2} \alpha^{-2} \omega_{, 1}\right)_{, 2}+\left(\rho^{2} \alpha^{-2} \omega_{, 2}\right)_{, 1}=f_{, 2},
$$

where derivatives are taken with respect to real coordinates $x^{a}$ and $f$ is a $\varphi$-independent function. One can consider (60) as an equation for $\omega$, in which $\varphi$ is a parameter, or one can formally solve (60) to obtain

$$
\rho^{2} \alpha^{-2} \omega_{, 1}-f=\gamma_{, 1}, \quad \rho^{2} \alpha^{-2} \omega_{, 1}=-\gamma_{, 2}
$$

where $\gamma$ is a new function. Let us treat (61) as equations for $\omega$ and $\rho^{2} \alpha^{-2}$ whereas $\gamma$ and $f$ are given. Then $\omega$ has to satisfy the first order linear equation

$$
\gamma_{, 2} \omega_{, 1}+\left(\gamma_{, 1}+f\right) \omega_{, 2}=0 .
$$

If it is solved function $\rho^{2} \alpha^{-2}$ is given by

$$
\frac{\rho^{2}}{\alpha^{2}}=\frac{\left(\gamma_{, 1}+f\right) \omega_{, 1}-\gamma_{, 2} \omega_{, 2}}{\omega_{, 1} \omega_{, 1}+\omega_{, 2} \omega_{, 2}}
$$

provided that the nominator and the denominator of the r. h. s. of (63) are both positive. Solving (62) with respect to $\omega$ is equivalent to finding integral lines of the vector field $v=\gamma_{, 2} \partial_{1}+\left(\gamma_{, 1}+f\right) \partial_{2}$ in $R^{2}$. The latter problem can be reduced to an ordinary differential equation with an arbitrary initial condition.

\section{Summary}

We have been considering initial constraints for the vacuum Einstein equations in the framework of the conformal approach. We assumed that metric can be put into form (13). One can relate with coordinates $\xi, \bar{\xi}, \varphi$ the Cauchy-Riemann operator $\partial$. The momentum constraint with $\tilde{H}=$ const splits into Eqs. (14) and (15) (Proposition 2.1). 
Equation (14) can be directly integrated with respect to $W$ giving formula (23). In the case of fields analytic in coordinate $\varphi$ or data with $\beta=0$ solutions of equation (15) can be also written as integrals of known functions (Propositions 2.2 and 2.3). In order to complete the construction of initial data one has still to solve the Lichnerowicz equation (4) for the conformal factor $\psi$. Its existence and uniqueness can be easily proved in some cases, e.g. if data $(g, K)$ are asymptotically flat, $\tilde{H}=0$ and the Ricci scalar of $g$ is nonnegative.

In Sect. 3 we propose a construction of maximal data with a reflection symmetry which, together with (49), implies existence of a horizon in the form of a marginally outer trapped surface (Proposition 3.1). As an example we present data obtained by a modification of the Kerr initial data.

Open Access This article is distributed under the terms of the Creative Commons Attribution 4.0 International License (http://creativecommons.org/licenses/by/4.0/), which permits unrestricted use, distribution, and reproduction in any medium, provided you give appropriate credit to the original author(s) and the source, provide a link to the Creative Commons license, and indicate if changes were made.

\section{References}

1. Choquet-Bruhat, Y.: General Relativity and Einstein's Equations. Oxford University Press, Oxford (2009)

2. Cook, G.B.: Initial data for numerical relativity. Living Rev. Relativ. 3, 2000-5 (2000). doi:10.12942/ 1rr-2000-5

3. Isenberg, J.: The Initial Value Problem in General Relativity. In: Ashtekar, A., Petkov, V. (eds.) Springer Handbook of Spacetime. Springer, Berlin (2014)

4. Brill, D.R., Lindquist, R.W.: Interaction energy in geometromechanics. Phys. Rev. 131, 471-476(1963)

5. Misner, C.W.: The method of images in geometrostatics. Ann. Phys. 24, 102-117 (1963)

6. Bowen, J.M., York Jr, J.W.: Time-asymmetric initial data for black holes and black hole collisions. Phys. Rev. D 21, 2047-2055 (1980)

7. Brandt, S.R., Brügmann, B.: A simple construction of initial data for multiple black holes. Phys. Rev. Lett. 78, 3606-3609 (1997)

8. Brandt, S.R., Seidel, E.: Evolution of distorted rotating black holes. III: initial data. Phys. Rev. D 54, 1403-1416 (1996)

9. Baker, J., Puzio, R.: New method for solving the initial value problem with application to multiple black holes. Phys. Rev. D 59, 044030 (1999)

10. Dain, S.: Initial data for a head-on collision of two Kerr-like black holes with close limit. Phys. Rev. D 64, 124002 (2001)

11. Conboye, R., Murchadha, N.Ó.: Potentials for transverse trace-free tensors. Class. Quantum Gravity 31, 085019 (2014)

12. Tafel, J., Jóźwikowski, M.: New solutions of initial conditions in general relativity. Class. Quantum Gravity 31, 115001 (2014)

13. Cantor, M., Brill, D.R.: The Laplacian on asymptotically flat manifolds and the specification of scalar curvature. Compos. Math. 43, 317-325 (1981)

14. Dain, S.: Trapped surfaces as boundaries for the constraint equations. Class. Quantum Gravity 21, 555-571 (2004)

15. Maxwell, D.: Solutions of the Einstein constraint equations with apparent horizon boundaries. Commun. Math. Phys. 253, 561-583 (2005)

16. Maxwell, D.: Rough solutions of the Einstein constraint equations. J. Reine Angew. Math. 590, 1-30 (2006)

17. Alcubierre, M.: Introduction to $3+1$ Numerical Relativity. Oxford Science Publications, Oxford (2008)

18. Baumgarte, T., Shapiro, S.: Numerical Relativity. Cambridge University Press, Cambridge (2010)

19. Choquet-Bruhat, Y., Isenberg, J., York Jr, J.W.: Einstein constraints on asymptotically Euclidean manifolds. Phys. Rev. D 61, 084034 (2000) 
20. Smarr, L.: Gauge condition, radiation formulae and the two black hole collision. In: Smarr, L. (ed.) Sources of Gravitational Radiation. Cambridge University Press, Cambridge (1979)

21. Thornburg, J.: Coordinates and boundary conditions for the general relativistic initial data problem. Class. Quantum Gravity 4, 1119-1131 (1987)

22. Boggess, A.: CR Manifolds and the Tangential Cauchy-Riemann Complex. Advanced Studies in Mathematics. CRC Press, London (1991)

23. Lewy, H.: An example of a smooth linear partial differential equation without solution. Ann. Math. 66, 155-158 (1957)

24. Lewandowski, J., Nurowski, P., Tafel, J.: Algebraically special solutions of the Einstein equations with pure radiation fields. Class. Quantum Gravity 8, 493-501 (1991)

25. Hörmander, L.: An introduction to complex analysis in several variables, 3rd edn. Elsevier Science Publishers B.V, North Holland (1990) 